\title{
Virus and Antibody Diagnostics for Swine Samples of the Dominican Republic Collected in Regions Near the Border to Haiti
}

\author{
A. Ventura, ${ }^{1}$ W. Gonzalez, ${ }^{1}$ R. Barrette, ${ }^{2}$ S. Swenson, ${ }^{3}$ A. Bracht, ${ }^{2}$ J. Rowland, ${ }^{2}$ A. Fabian, ${ }^{2}$ \\ K. Moran, ${ }^{2}$ F. Mohamed, ${ }^{2}$ E. O'Hearn, ${ }^{2}$ M. Jenkins-Moore, ${ }^{3}$ D. Toms, ${ }^{3}$ J. Shaw, ${ }^{4}$ P. Morales, ${ }^{4}$ \\ D. Pyburn, ${ }^{5}$ C. Carrillo, ${ }^{2}$ G. Mayr, ${ }^{2}$ M. McIntosh, ${ }^{2}$ and M. Deng ${ }^{2}$ \\ ${ }^{1}$ Ministry of Agriculture, Santo Domingo, Dominican Republic \\ ${ }^{2}$ USDA, APHIS, VS, NVSL, FADDL, Greenport, NY 11944, USA \\ ${ }^{3}$ USDA, APHIS, VS, NVSL, Ames, IA 50010, USA \\ ${ }^{4}$ USDA, APHIS, IS, US Embassy, Santo Domingo, Dominican Republic \\ ${ }^{5}$ USDA, APHIS, VS, Swine Health Program, Des Moines, IA, USA
}

Correspondence should be addressed to M. Deng; ming.y.deng@aphis.usda.gov

Received 11 July 2012; Accepted 9 August 2012

Academic Editors: J. M. Casasnovas, R. G. Dietzgen, and J. Tozser

Copyright (C) 2013 A. Ventura et al. This is an open access article distributed under the Creative Commons Attribution License, which permits unrestricted use, distribution, and reproduction in any medium, provided the original work is properly cited.

The Dominican Republic (DR) and Haiti share the island of Hispaniola, and reportable transboundary animal diseases have been introduced between the two countries historically. Outbreaks of severe teschovirus encephalomyelitis in pigs began occurring in Haiti in February 2009, and a field and laboratort study in April 2010 indicated that the teschovirus disease is prevalent in many regions in Haiti including areas near the border with DR and that other viral disease agents, including CSF virus (CSFV), porcine circovirus type 2 (PCV-2), porcine reproductive and respiratory syndrome virus (PRRSV), and swine influenza virus (SIV), are present in the swine population in these regions. The purpose of this study was to evaluate the introduction of teschovirus encephalomyelitis from Haiti to DR and to identify the other viral disease agents present in the swine population in regions of DR near the border with Haiti. Six of 7 brains and 6 of 7 spinal cords collected from pigs with central nervous system (CNS) signs were positive in reverse transcription-polymerase chain reaction for PTV. Genome sequencing on the Dominican PTV and phylogenetic analysis on the polyprotein of PTV strains indicate that the sequence of the Dominican PTV is $99.1 \%$ identical to the Haitian isolate and closely related to other PTV-1 strains in the world. Among 109 serum samples tested, 65 (59.6\%) were positive for antibodies to PCV-2, and 51 (46.8\%) were positive for antibodies to CSFV. Fifty-four of the 109 serum samples were tested for antibodies to other agents. Among the 54 samples, 20 (37.0\%) were seropositive to PTV-1, 17 (31.5\%) tested seropositive to SIV H3N2, 12 (22.2\%) were seropositive to SIV H1N1, and 1 (1.9\%) was seropositive to PRRSV.

\section{Introduction}

The Dominican Republic (DR) and Haiti share the island of Hispaniola, the second largest island in the Caribbean. Historically, reportable transboundary animal diseases have been introduced between the two countries. In 1978, an outbreak of African swine fever (ASF) was detected in DR, and the source of the outbreak was potentially linked to food waste from an airport that was fed to swine [1]. In order to prevent the introduction of the disease, the Haitian government ordered a preventive slaughter of all swine in a 15-km area along the border with DR [1]. Despite this effort, ASF was detected in Haiti and spread rapidly throughout the country in late 1978 [1]. ASF along with classical swine fever (CSF), which had been endemic there since about 1920, were both eradicated by the slaughter of the entire swine population in the Hispaniola in 1984 [2]. CSF re-entered Haiti with a severe outbreak of unknown origin throughout the country in 1996 [3]. In March 1997, CSF spread from Haiti to DR causing 22 outbreaks that year and 232 outbreaks in 1998 [3]. Currently, CSF is considered endemic in both countries of the Hispaniola [2]. 
Teschovirus encephalomyelitis of pigs is caused by porcine teschovirus (PTV), a member of the genus Teschovirus, the family Picornaviridae [4]. There are at least eleven distinct serotypes of PTV, PTV-1 through PTV-11. Some virulent strains of PTV-1 cause severe encephalomyelitis, and other strains of PTV-1 as well as other PTV serotypes can cause milder forms of the disease or inapparent infections in pigs [4-6]. Outbreaks of severe teschovirus encephalomyelitis in pigs began occurring in Haiti in February 2009, and PTV1 was isolated from brain samples of sick pigs [7]. Results of sequence and phylogenetic analyses on the polyprotein of PTV strains indicate that the Haitian isolate is most closely related to other PTV-1 strains, including the strain Konratice which was isolated in Czechoslovakia from pigs with porcine viral encephalomyelitis (Teschen disease) [7]. One of two pigs inoculated with the Haitian PTV-1 isolate showed typical clinical signs of teschovirus encephalomyelitis including paralysis of the hind quarters 32 days postinoculation at the National Veterinary Service Laboratories (NVSL), Animal and Plant Health Inspection Services (APHIS), the United States Department of Agriculture (USDA), Ames, IA (John J. Schiltz et al. of NVSL, unpublished results). There are currently no vaccines commercially available for the disease [4]. In April 2010, a field and laboratory study was conducted collaboratively by the Haitian government, USDA/APHIS, Food and Agriculture Organization (FAO) of the United Nations, and the International Institute for Cooperation on Agriculture (IICA) on thirty-five swine farms located in multiple regions in Haiti including those close to the border with DR. Results of this study indicate that teschovirus encephalomyelitis is prevalent in many regions in Haiti including areas near the border with DR and that other viral disease agents, including CSF virus (CSFV), porcine circovirus type 2 (PCV-2), porcine reproductive and respiratory syndrome virus (PRRSV), and swine influenza virus (SIV) H3N2 and H1N1, are present in the Haitian swine population (Rodney Jacques-Simon, Max Millien, Keith Flanagan, John Shaw, Paula Morales, Julio Pinto, David Pyburn, Wendy Gonzalez, Angel Ventura, Thierry Lefrancois, Jennifer Pradel, Sabrina Swenson, Melinda JenkinsMoore, Dawn Toms, Matthew Erdman, Linda Cox, Alexa J. Bracht, Andrew Fabian, Fawzi M. Mohamed, Karen Moran, Emily O'Hearn, Consuelo Carrillo, Gregory Mayr, William White, Samia Metwally, Michael T. McIntosh, and Mingyi Deng: A field and laboratory investigation on viral diseases of swine in the Republic of Haiti, submitted for publication). In 2010, suspected cases of teschovirus encephalomyelitis were reported from regions of DR near the border to Haiti. The purpose of this study was to evaluate the introduction of teschovirus encephalomyelitis from Haiti to DR and to identify the other viral disease agents present in the swine population in regions of DR near the border with Haiti.

\section{Materials and Methods}

2.1. Sample Collections. Samples were collected from thirtysix swine premises in DR in August 2010. All these premises are located in regions near the border of Haiti. These premises were utilized for raising backyard pigs. One-hundred and nine sera were collected from pigs including those clinically suspected of having PTV infection as well as apparently healthy pigs. On average, three serum samples were taken from each premise. Whenever possible, pigs with central nervous system (CNS) signs, such as incoordination, opisthotonus, and paralysis of hindquarters, were euthanized and necropsied. Brains, spinal cords, and tonsils from seven necropsied animals (pig no. 57, 62, 69, 73, 91, 92, and 93) were collected. During sample collections, animal information including vaccination status, movement, and disease history was obtained from the owner of each premise. The 109 serum samples were split into two subsets: one subset of 109 and another of 54 because only 54 samples had adequate volume for the second subset. The subset of 109 sera and all tissue samples were sent on ice for laboratory tests to the Foreign Animal Disease Diagnostic Laboratory (FADDL) of the NVSL, USDA on Plum Island, NY. The subset of 54 serum samples was sent on ice to the NVSL in Ames, IA.

2.2. Sample Preparation and Virus Isolation. A $10 \%$ tissue homogenate was prepared from brain, spinal cord, and tonsil samples in Eagle's minimal essential medium (EMEM) (Lonza, Walkersvelle, MD, USA) supplemented with $4 \%$ fetal bovine serum (FBS) (Lonza) using a Mixer Mill (MM300, Retsch Inc, Newtown, PA, USA) at a vibrational frequency of 18 cycles per second for a total of two minutes. All homogenates were clarified by centrifugation at $2,500 \times \mathrm{g}$ for 10 minutes and then filtered through $0.45-\mu \mathrm{m}$ cellulose acetate filters (GE Water and Process Technologies, Trevose, PA, USA). For virus isolation, a volume of $0.5 \mathrm{~mL}$ of tissue homogenate or serum was inoculated onto monolayer cultures of swine kidney (SK-6), IBRS-2, and Vero cells grown in $25 \mathrm{~cm}^{2}$ cell culture flasks. The inoculum was adsorbed for 60 minutes at $37^{\circ} \mathrm{C}$ and $5 \mathrm{~mL}$ of EMEM with $4 \%$ FBS was then added to each flask. The flasks were incubated at $37^{\circ} \mathrm{C}$ and observed daily for cytopathic effect (CPE). When no CPE was observed after three days of incubation, the flasks were frozen and thawed, and a volume of $0.5 \mathrm{~mL}$ of lysate of each flask was inoculated into a new flask of each cell line described above. Cultures positive for CPE were frozen at $-70^{\circ} \mathrm{C}$ for further analysis in virus identification. For isolation of CSFV, cultures of SK-6 cells negative for CPE were tested for CSFV antigens with the avidin biotin complex $(\mathrm{ABC})$ immunohistochemistry assay described below.

2.3. Detection of CSFV Antigens. Tonsils and cultures of SK6 cells negative for CPE were tested for CSFV antigens with the $\mathrm{ABC}$ immunohistochemistry assay using the VECTASTAIN ABC-AP Kit (Vector Laboratories Inc., Burlingame, CA, USA) and monoclonal antibody V3 (Cedi Diagnostics, Lelystad, the Netherlands) against the glycoprotein 55 of CSFV according to the manufacturers' instructions.

2.4. Nucleic Acid Extraction. RNA was extracted from $140 \mu \mathrm{L}$ of each tissue homogenate of brain, spinal cord, tonsil, and serum samples with the RNeasy Mini Kit (Qiagen, Inc., 
Valencia, CA, USA) following the manufacturer's instructions. RNA from each sample was eluted in $40 \mu \mathrm{L}$ of RNasefree water and stored at $-70^{\circ} \mathrm{C}$ until RT-PCR was performed.

2.5. RT-PCR for PTV and Real-Time RT-PCR for CSFV. For the detection of PTV, two RT-PCR assays were conducted on RNA of brain and spinal cord samples as described previously [7] following procedures of Zell et al [8]. The first assay was a nested RT-PCR for the detection of PTV-1 through PTV-11. The second assay was specific for PTV-1. For the detection of CSFV, a TaqMan real-time RT-PCR was conducted on RNA of serum and tonsil samples according to Risatti et al. [9] as described previously [7].

2.6. Detection of Antibodies to CSFV. The HerdCheck CSFV Antibody Test Kit (IDEXX Laboratories, Inc., Westbrook, ME) was used for serum samples according to the manufacturer's instructions. A sample was considered positive for the presence of antibodies to a pestivirus if its blocking percentage was $40 \%$ or greater. An immunoperoxidase (IP) test was further performed to determine if the positive result is due to the presence of antibodies to CSFV $[10,11]$. Briefly, CSFV-infected and uninfected swine kidney (SK-6) cells prepared in microtiter plates were fixed and used in the IP test. After incubation with test serum, bound antibodies were reacted with a protein G-horseradish peroxidase conjugate (Invitrogen Corporation, Carlsbad, CA) followed by the addition of the AEC substrate (Vector Laboratories, Inc., Burlingame, CA). The plates were washed with PBS, pH 7.2 containing $0.05 \%$ Tween 20 and read using an inverted light microscope. A positive reaction was indicated by the presence of a red color in the cytoplasm of the CSFV-infected cells.

2.7. Detection of Antibodies to PTV-1. Porcine kidney (PK15) cells or primary swine kidney (pSK) grown on coverslips in Leighton tubes (Bellco, Vineland, NJ, USA) and were inoculated with PTV-1 Haiti/2009 (1.5 mL of virus dilution per tube) and incubated at $37^{\circ} \mathrm{C}$ overnight. The coverslips were fixed in acetone for 5-10 minutes and air dried. A volume of $200 \mu \mathrm{L}$ of each serum sample diluted $1: 20$ in PBS; $\mathrm{pH} 7.2$ was added onto a coverslip and incubated at $37^{\circ} \mathrm{C}$ for 30 minutes in a humid chamber. The coverslips were rinsed in PBS, pH 7.2 and soaked in PBS for 5 minutes. A volume of $200 \mu \mathrm{L}$ of a $1: 75$ dilution of goat anti-pig IgG conjugated with fluorescein isothiocyanate (FITC) was added onto each coverslip and incubated at $37^{\circ} \mathrm{C}$ for 30 minutes in a humid chamber. The coverslips were rinsed in PBS, $\mathrm{pH}$ 7.2 , soaked in PBS for 5 minutes, rinsed in reverse osmosis water and allowed to air dry. After drying, the coverslips were mounted on microscope slides with a mounting medium containing 50\% glycerin and 50\% PBS and examined under a fluorescence microscope. PTV-1 infected cells on slides with serum samples positive for antibody to the virus appeared bright green on a dark background.

2.8. Detection of Antibodies to PCV-2, PRRSV, and Pseudorabies Virus (PRV). SERELISA PCV2 Ab Mono Blocking Detection Kit (Synbiotics Corporation, Kansas City, MO,
USA), IDEXX PRRS X3 Ab Test (IDEXX Laboratories, Inc.) and IDEXX PRV/ADV gB Ab Test (IDEXX Laboratories, Inc.) were used for the detection of antibodies to PCV-2, PRRSV and PRV in serum samples, respectively according to the manufacturers' instructions.

2.9. Detection of Antibodies to SIV. The National Veterinary Services Laboratories Standard Operating Protocol for SIV hemagglutination-inhibition (HI) was used for the detection of anti-H1N1 and anti-H3N2 antibodies [12]. Briefly, serum samples were first treated with a receptor destroying enzyme (RDE) (Bio Whittacker, Walkersville, MD, USA) at $37^{\circ} \mathrm{C}$ for $12-18$ hours, inactivated at $56^{\circ} \mathrm{C}$ for $30 \pm 5$ minutes after adding $0.15 \mathrm{~mL}$ of $2.5 \%$ sodium citrate solution, and then treated with packed/washed turkey red blood cells for 30 minutes at room temperature. Each sample was then serially 2 -fold diluted in PBS, pH 7.2 in a V-bottom 96-well micro-titer plate and tested for its $\mathrm{HI}$ activity against 4-8 hemagglutination (HA) units of $\mathrm{H} 1$ (H1N1 A/SW/NC/2001) or H3 (H3N2 A/SW/TX/4199/98) reference strains of SIV. The presence of $\mathrm{HI}$ antibody in each sample against $\mathrm{H} 1$ or $\mathrm{H} 3$ SIV was determined by adding $0.5 \%$ rooster or turkey RBC and incubating for 30-60 minutes at room temperature. HI antibody titer of each sample was interpreted as the reciprocal of the highest dilution in which no hemagglutination was observed. Samples with no HI activity at 1:10 dilution were considered negative.

2.10. Sequence Analysis. For the characterization of the genome of the PTV identified in this investigation, overlapping cDNA fragments of the PTV genome spanning the polyprotein coding region were amplified by RT-PCR using primers previously designed for the genome of PTV1, Haiti/2009 [7]. RT-PCR products were subjected to Sanger dideoxy-sequencing using a BigDye Terminator v3.1 Cycle Sequencing Kit (Applied Biosystems, Carlsbadm, CA, USA). Sequencing reactions were purified using the Agencourt CleanSEQ system (Beckman Coulter, Inc., Brea, CA, USA) and analyzed on a 3730XL DNA Analyzer (Applied Biosystems). Sequence contigs were read and assembled using Sequencher (Gene Codes Corporation, Ann Arbor, MI, USA).

2.11. Phylogenetic Analysis. Phylogenetic analysis was performed by alignment of the nucleotide sequence of the PTV obtained in this study with those of other teschoviruses using the version 1.4 of ClustalW software [13]. Sequences were trimmed, edited, and exported to PHYLIP format using BioEdit software (version 7.0.5.3) [14]. Phylogenetic analysis and assembly of the tree for PTV was performed using PHYLIP software package version 3.69 (Available at: http://evolution.genetics.washington.edu/phylip /getme.html, accessed March 22, 2012) for analyzing the complete polyprotein coding region of teschoviruses $[15,16]$. The bootstrap consensus trees were inferred from 1,000 replicates with calculation of the distance matrix based on nucleotide sequence. The output trees were then analyzed using the Neighbor-joining method to produce a final consensus tree. 
Phylogenetic tree graphics were produced using DRAWGRAM tool (Available at: http://evolution.genetics.washington.edu/phylip/doc/drawgram.html, accessed March 22, 2012). Output tree was annotated to include percent consensus out of 1,000 trees with Adobe Illustrator CS4 version 14.0.0 (Adobe Systems Inc., San Jose, CA, USA).

2.12. Histopathological Analysis. Brain tissues were fixed in $10 \%$ neutral buffered formalin, were paraffin-embedded, sectioned at four microns, mounted on glass slides and stained with hematoxylin and eosin as described by Fischer et al. [17].

\section{Results}

3.1. Detection of Viruses. Brains and spinal cords from all seven necropsied animals were tested by RT-PCR for PTV and those from six animals (pig no. 57, 62, 69, 91, 92, and 93) were positive in the RT-PCR. For the characterization of PTV in these samples, a RT-PCR for the amplification of the polyprotein coding region of the viral genome was conducted individually on the six RT-PCR positive brain samples initially. Only the brain sample from pig no. 91, however, yielded satisfactory amplicon and thus genome sequencing was completed for the polyprotein coding region of the PTV in this sample. The result of phylogenetic analysis on the polyprotein of the PTV stains indicate that the sequence of the Dominican PTV is $99.1 \%$ identical to the Haitian isolate and closely related to other PTV-1 strains (Figure 1). The Dominican PTV was designated as PTV1/Dominican Republic/2010. The nucleotide sequence of this virus was submitted to GenBank with accession no. JQ808131. No viable PTV and other viruses were isolated in cell cultures from any of the brain and spinal cord samples.

Tonsil samples from all seven necropsied animals were negative in the real-time RT-PCR, virus isolation, and the $\mathrm{ABC}$ assay for CSFV. The subset of 109 serum samples were also tested by the real-time RT-PCR for CSFV and none were positive for CSFV.

3.2. Histopathological Analysis. In the histopathological analysis, the cerebrum, cerebellum, and brain stem of each of seven brain samples were examined. The most significant microscopic finding from these samples was multifocal, nonsuppurative encephalomyelitis as observed in Haitian samples from pigs affected by teschovirus encephalomyelitis as described previously [7].

3.3. Detection of Antibodies to Viruses in Sera. The subset of 109 serum samples were tested by immunological assays for antibodies to PCV-2 and CSFV as described in Section 2 at FADDL, Plum Island, NY. Among these serum samples, $65(59.6 \%)$ were positive for antibodies to PCV-2, and 51 (46.8\%) were positive for antibodies to CSFV (Figure 2). The PCV-2 and CSFV sero-positives were scattered in all study areas (data not shown). Of the 51 serum samples positive for antibodies to CSFV, 26 (51.0\%) were collected from CSF-vaccinated pigs and $25(49.0 \%)$ from nonvaccinated pigs. Among 58 samples negative for antibodies to CSFV, $8(13.8 \%)$ were collected from CSF-vaccinated pigs and 50 (86.2\%) from nonvaccinated pigs.

The subset of 54 sera of the 109 serum samples were tested for antibodies to other viral agents at NVSL-Ames, IA. Of these 54 samples, the numbers of samples positive for antibodies to various viral agents were: PTV-1, 20 (37.0\%); SIV H3N2, 17 (31.5\%); SIV H1N1, 12 (22.2\%); and PRRSV, $1(1.9 \%)$. All the 54 samples were negative for antibodies to PRV (Figure 2). Among the 20 samples positive for antibodies to PTV-1, 7 (35.0\%) were collected from euthanized sick pigs with CNS signs and 13 (65.0\%) from clinically healthy pigs; $15(75.0 \%)$ were positive and $5(25 \%)$ were negative for antibodies to PCV-2. Among seven euthanized and PTV-1 antibody-positive sick pigs, 3 were positive for antibodies to PCV-2.

\section{Discussion}

Results of this study indicate that teschovirus encephalomyelitis has spread to the DR in regions close to the border with Haiti, and that other viral disease agents, that is, CSFV, PCV2, PRRSV, and SIV, are present in the swine population of these regions.

Genome sequencing and phylogenetic analysis on PTV stains indicate that the sequence of the Dominican PTV is $99.1 \%$ identical to the Haitian isolate and closely related to other PTV-1 strains. As described previously [7], among these PTV-1 strains, Konratice (AF231767) was isolate in Czechoslovakia from pigs with porcine viral encephalomyelitis (Teschen disease) [18]. PTV-1 Strains Konratice and Bozen (AF231767) produced a lethal paralytic disease by intramuscular inoculation in pigs [19], and strain F65 (AJ011380) produced clinical signs resulting from neuroinvasiveness when inoculated into specific-pathogen free pigs [20]. Surprisingly, no viable PTV was isolated in cell cultures from any of the brains and spinal cords of the seven euthanized sick pigs with CNS signs while brain and spinal cord samples of six of these animals were positive in RTPCR for PTV. The negative results in virus isolation for the RT-PCR-positive samples are likely due to virus inactivation during collection, storage and international transportation of the samples cause by hot summer temperatures.

The evaluation of the introduction of PTV from Haiti to DR was the main objective of this study but the subset of 109 serum samples was not first tested for antibodies to PTV-1 partially due to the logistics of sample submission. As described in Materials and Methods, the 109 sera were split into two subsets: one subset of 109 and another of 54 . The subset of 109 sera and brains, spinal cords and tonsils from seven necropsied pigs were submitted to FADDL on Plum Island, NY for the detection of viruses and antibodies to CSFV and PCV-2. The subset of 54 serum samples was sent to the NVSL in Ames, IA for the detection of antibodies to PTV-1, SIV, PRRSV and PRV. The availability of laboratory assays in FADDL and the NVSL, Ames is different from each other and efforts are always made to avoid transferring samples from the bio-containment of FADDL on Plum Island 


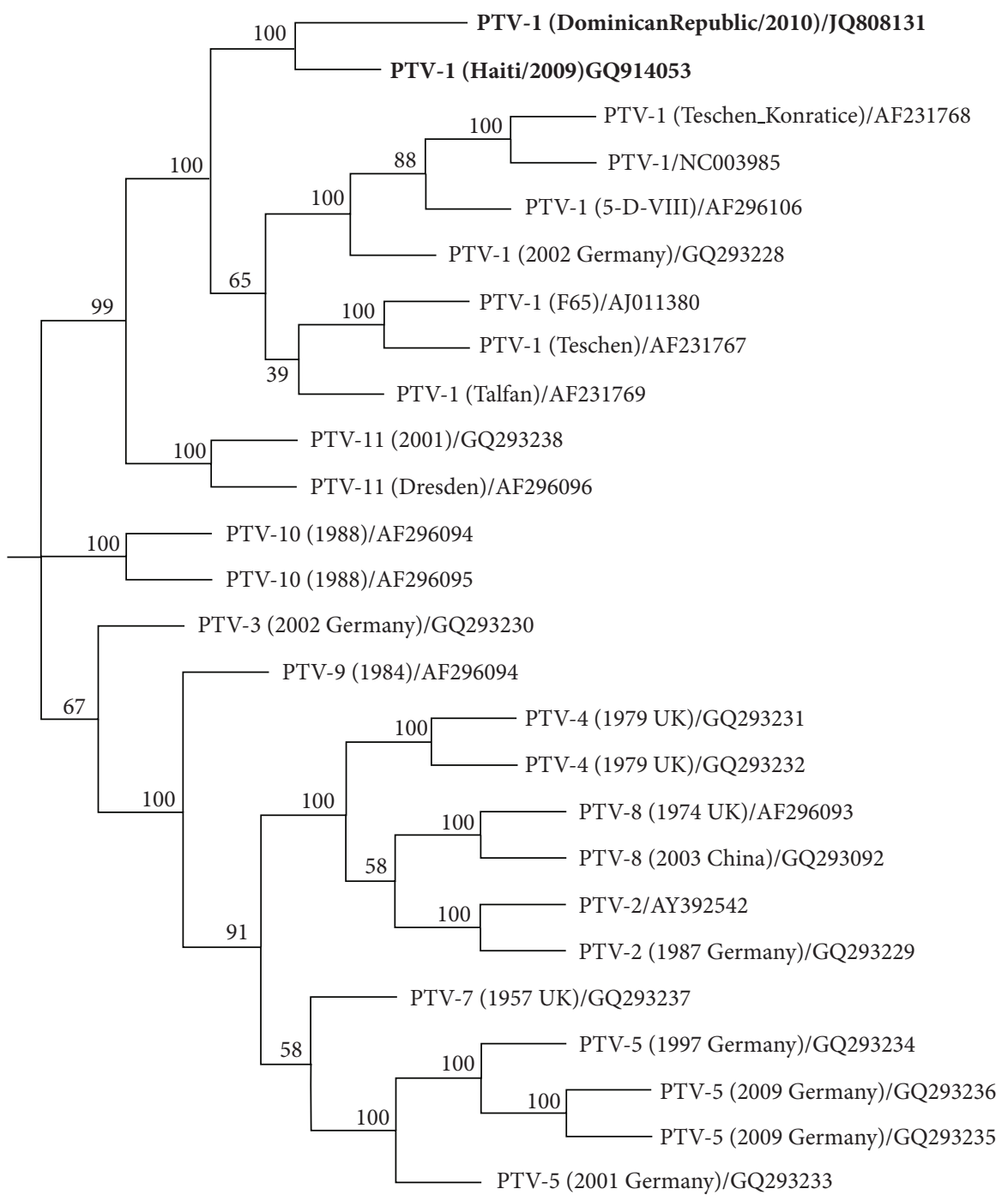

FIGURE 1: Phylogenetic analysis of the coding sequence for polyprotein of the porcine teschovirus (PTV) strains. The PTV detected and characterized from the brain of a sick pig with central nervous system signs (pig no. 91) of Dominican Republic is represented in bold along with PTV-1, Haiti/2009. Bootstrap consensus trees were inferred from 1,000 replicates with calculation of the distance matrix based on nucleotide sequence. The output trees were analyzed using the Neighbor-joining method to produce the final consensus tree. Percent consensus out of 1,000 trees is indicated at branch nodes. Nucleotide sequence of the Dominican Republic PTV was compared with sequences of 25 teschovirus Genbank accessions. Genbank accession numbers are identified in the leaf descriptors on the consensus tree.

to anywhere on the mainland of the United States. Thus, the subset of 109 serum samples was not shipped to the NVSL in Ames, IA for tests on antibodies to PTV-1 but the subset of 54 sera was tested for at the NVSL. The authors believe that the results from the 54 serum samples for the detection of antibodies PTV-1 and other viruses could serve the purpose of the study.

In the study, two viral agents (PTV and CSFV) were selected for screening in samples collected (PTV in brains and spinal cords; CSFV in sera and tonsils), while antibodies to several other viruses, such as PCV-2, PRRSV, SIV, and PRV were tested in sera. We did these because of certain considerations. First, pigs in suspected cases showed CNS signs and these signs are consistent with infections by PTV or CSFV. Second, laboratory assays for these viruses are readily available. Third, as for other viral agents, testing for antibodies to the agents in sera may be more effective to demonstrate the prevalence of these agents in the swine population than testing viral agents themselves which would not be detectable at all the time.

PTV infections often do not produce clinical signs and the seroprevalence of PTV-1 may exceed $60 \%$ in health pig populations in Central Europe [4]. High-virulent strains of PTV were not reported in the Western hemisphere until the outbreak in Haiti. Factors responsible for the expression of the severe teschovirus encephalomyelitis in the Hispaniola are not known. We speculated that there may be other disease agents present in the swine population in addition to CSFV and PTV, such as PCV-2 and PRRSV, that the immunosuppressive effect of these agents may have facilitated 


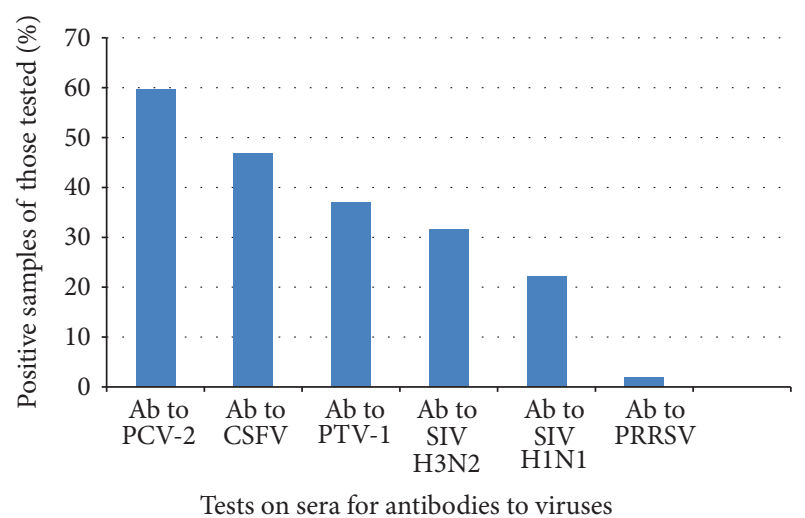

FIGURE 2: Results of testing serum samples collected from pigs in regions of Dominican Republic near the border to Haiti for the presence of antibodies (Ab) to porcine circovirus type 2 (PCV2), classical swine fever virus (CSFV), porcine teschovirus type 1 (PTV-1), swine influenza virus (SIV) H3N2, SIV H1N1 and porcine reproductive and respiratory syndrome virus (PRRSV). All serum samples tested were negative in the IDEXX PRV/ADV gB Ab Test for antibodies to pseudorabies virus. One hundred and nine serum samples were tested for antibodies to PCV-2 and CSFV, and 54 replicates of the 109 serum samples were tested for antibodies to other viral agents described.

the expression of PTV, and that adding commercially available vaccines for these agents to the on-going CSF vaccine program may be beneficial to the control of teschovirus disease. In addition, although other viral agents for swine, including SIV and PRV did not appear as an issue in DR, information on their presence in the country was not known. Thus, we tested antibodies to PCV-2, PRRSV, SIV, and PRV in sera together with other laboratory analyses.

In this study, among 20 serum samples positive for antibodies to PTV-1, 15 (75.0\%) were also positive for antibodies to PCV-2. This result is similar to what was obtained in analyzing serum samples collected from Haitian swine population: $64.8 \%$ of PTV-1 antibody-positive pigs were also positive for antibodies to PCV-2 (Rodney JacquesSimon, Max Millien, Keith Flanagan, John Shaw, Paula Morales, Julio Pinto, David Pyburn, Wendy Gonzalez, Angel Ventura, Thierry Lefrancois, Jennifer Pradel, Sabrina Swenson, Melinda Jenkins-Moore, Dawn Toms, Matthew Erdman, Linda Cox, Alexa J. Bracht, Andrew Fabian, Fawzi M. Mohamed, Karen Moran, Emily O'Hearn, Consuelo Carrillo, Gregory Mayr, William White, Samia Metwally, Michael T. McIntosh, and Mingyi Deng: A field and laboratory investigation on viral diseases of swine in the Republic of Haiti, submitted for publication). Results from these studies indicate that many of the pigs in Haiti and DR had a history of infection by both PTV-1 and PCV-2, but whether there is a close relationship between these two agents in the swine population of the two countries remains uncertain. Research has shown that PCV-2 vaccines are effective in the control of PCV associated diseases. To determine the effectiveness of a commercial PCV-2 vaccine (Ingelvac CircoFLEX, Boehringer Ingelheim) for improving the immunity of the swine population in Haiti and DR and thus reducing the occurrence of the teschovirus disease, a field trial on the vaccine is currently being conducted in Haiti.

The fact that, of the 20 serum samples positive for antibodies to PTV-1, 7 (35.0\%) were collected from sick and $13(65.0 \%)$ were from clinically healthy pigs, indicates that many pigs developed immunity to the virus without developing clinical disease.

The fact that twenty-five (49.0\%) of 51 CSF seropositive samples were collected from nonvaccinated pigs indicates CSFV circulation in the Dominican swine population. Only a small portion (13.8\%) of serum samples negative for antibodies to CSFV were collected from CSF-vaccinated pigs. This is in contrast with the finding from Haitian swine, where $44.6 \%$ of serum samples negative for antibodies to CSFV were from CSF-vaccinated pigs (Rodney Jacques-Simon, Max Millien, Keith Flanagan, John Shaw, Paula Morales, Julio Pinto, David Pyburn, Wendy Gonzalez, Angel Ventura, Thierry Lefrancois, Jennifer Pradel, Sabrina Swenson, Melinda JenkinsMoore, Dawn Toms, Matthew Erdman, Linda Cox, Alexa J. Bracht, Andrew Fabian, Fawzi M. Mohamed, Karen Moran, Emily O'Hearn, Consuelo Carrillo, Gregory Mayr, William White, Samia Metwally, Michael T. McIntosh, and Mingyi Deng: A field and laboratory investigation on viral diseases of swine in the Republic of Haiti, submitted for publication). Negative test results for antibodies to CSFV in serum samples from CSF-vaccinated pigs may be indicative of less optimal quality of the vaccination work in the sampling regions, possibly due to inappropriate handling or administration of the vaccine.

\section{Acknowledgments}

The authors thank Rafael Nunez, the Ministry of Agriculture of Dominican Republic for his support to this project and Persio Almanzar, Ausberto Rodriguez, Simon de Leon, Carlixto Encarnacion, Corporino Novas, Julia Vargas, and Felix del Orbe for their assistance in sample collection and processing. The authors also thank Elizabeth Lautner, Paul Hauer, Beverly Schmitt, William White, Samia Metwally, Tami Beach, Annette Olson, Linda Cox, Heather Petrowski, and many others in USDA/APHIS/NVSL and the National Veterinary Diagnostic Laboratory of Haiti who were involved in sample collection and diagnosis. Ethan Macnow of Plum Island Animal Disease Center provided assistance in preparing the graphs used in the paper.

\section{References}

[1] Interamerica Institute for Cooperation on Agriculture, African Swine Fever Eradication and Swine Industry Development in Haiti, IICA, Port-au-Price, Haiti, 1981.

[2] FAO, OIRSA, OIPORC, and OIE, "Continental plan for the eradication of classical swine fever (CSF) from Americas," 2009, http://www.rlc.fao.org/es/prioridades/transfron/ppc/pdf/mpin gl.pdf.

[3] Food and Agriculture Oranization of the United Nations, "The Classical swine fever eradication plan for the Americas," 2000, http://www.rlc.fao.org/es/prioridades/transfron/ppc/pdf/18csf .pdf. 
[4] World Organization for Animal Health (OIE), "Teschovirus encephalomyelitis," in Manual of Diagnostic Tests and Vaccines For Terrestrial Animals, pp. 1146-1151, OIE, Paris, France, 2008.

[5] D. S. Bangari, R. M. Pogranichniy, T. Gillespie, and G. W. Stevenson, "Genotyping of Porcine teschovirus from nervous tissue of pigs with and without polioencephalomyelitis in Indiana," Journal of Veterinary Diagnostic Investigation, vol. 22, no. 4, pp. 594-597, 2010.

[6] The Center for Food Security and Public Health of Iowa State University, "Teschovirus encephalomyelitis and porcine teschovirus infection,” 2009, http://www.cfsph.iastate.edu/ Factsheets/pdfs/enterovirus_encephalomyelitis.pdf.

[7] M. Y. Deng, M. Max, R. Jacques-Simon et al., "Diagnosis of porcine teschovirus encephalomyelitis in the Republic of Haiti," Journal of Veterinary Diagnostic Investigation, vol. 24, no. 4, pp. 671-678, 2012.

[8] R. Zell, A. Krumbholz, A. Henke et al., "Detection of porcine enteroviruses by nRT-PCR: differentiation of CPE groups I-III with specific primer sets," Journal of Virological Methods, vol. 88, no. 2, pp. 205-218, 2000.

[9] G. R. Risatti, J. D. Callahan, W. M. Nelson, and M. V. Borca, "Rapid detection of classical swine fever virus by a portable real-time reverse transcriptase PCR assay," Journal of Clinical Microbiology, vol. 41, no. 1, pp. 500-505, 2003.

[10] A. Afshar, G. C. Dulac, and A. Bouffard, "Application of peroxidase labelled antibody assays for detection of porcine IgG antibodies to hog cholera and bovine viral diarrhea viruses," Journal of Virological Methods, vol. 23, no. 3, pp. 253-262, 1989.

[11] G. C. Saunders and M. E. Wilder, "Disease screening with enzyme labeled antibodies," Journal of Infectious Diseases, vol. 129, no. 3, pp. 362-364, 1974.

[12] T. Beach, National Veterinary Services LaboraTories Standard Operating Protocol SOP-BPA-0801.05, HemagglutinationInhibition Test For Detection of Antibodies to Swine Influenza Virus, H1N1 or H3N2, National Veterinary Services Laboratories, Ames, Iowa, USA, 2010.

[13] J. D. Thompson, D. G. Higgins, and T. J. Gibson, "CLUSTAL $\mathrm{W}$ : improving the sensitivity of progressive multiple sequence alignment through sequence weighting, position-specific gap penalties and weight matrix choice," Nucleic Acids Research, vol. 22, no. 22, pp. 4673-4680, 1994.

[14] T. A. Hall, "BioEdit: a user-friendly biological sequence alignment editor and analysis program for Windows 95/98/NT," Nucleic Acids Symposium Series, vol. 41, pp. 95-98, 1999.

[15] J. Felsenstein, "Confidence limits on phylogenies: an approach using the bootstrap," Evolution, vol. 39, no. 4, pp. 783-791, 1985.

[16] K. Tamura, M. Nei, and S. Kumar, "Prospects for inferring very large phylogenies by using the neighbor-joining method," Proceedings of the National Academy of Sciences of the United States of America, vol. 101, no. 30, pp. 11030-11035, 2004.

[17] A. H. Fischer, K. A. Jacobson, J. Rose J, and R. Zeller, "Hematoxylin and eosin staining of tissue and cell sections," Cold Spring Harb Protoc, no. 5, 2008.

[18] D. C. Blood and V. P. Studdert, Saunders Comprehensive Veterinary Dictionary, WB Saunders, London, UK, 2nd edition, 1999.

[19] A. Mayr, "Degree of variation of the virus of Teschen disease and relationship to other enteroviruses of swine," Bulletin de l'Office International des Epizooties, vol. 56, pp. 106-110, 1961.

[20] M. Doherty, D. Todd, N. McFerran, and E. M. Hoey, "Sequence analysis of a porcine enterovirus serotype 1 isolate: relationships with other picornaviruses," Journal of General Virology, vol. 80, no. 8, pp. 1929-1941, 1999. 

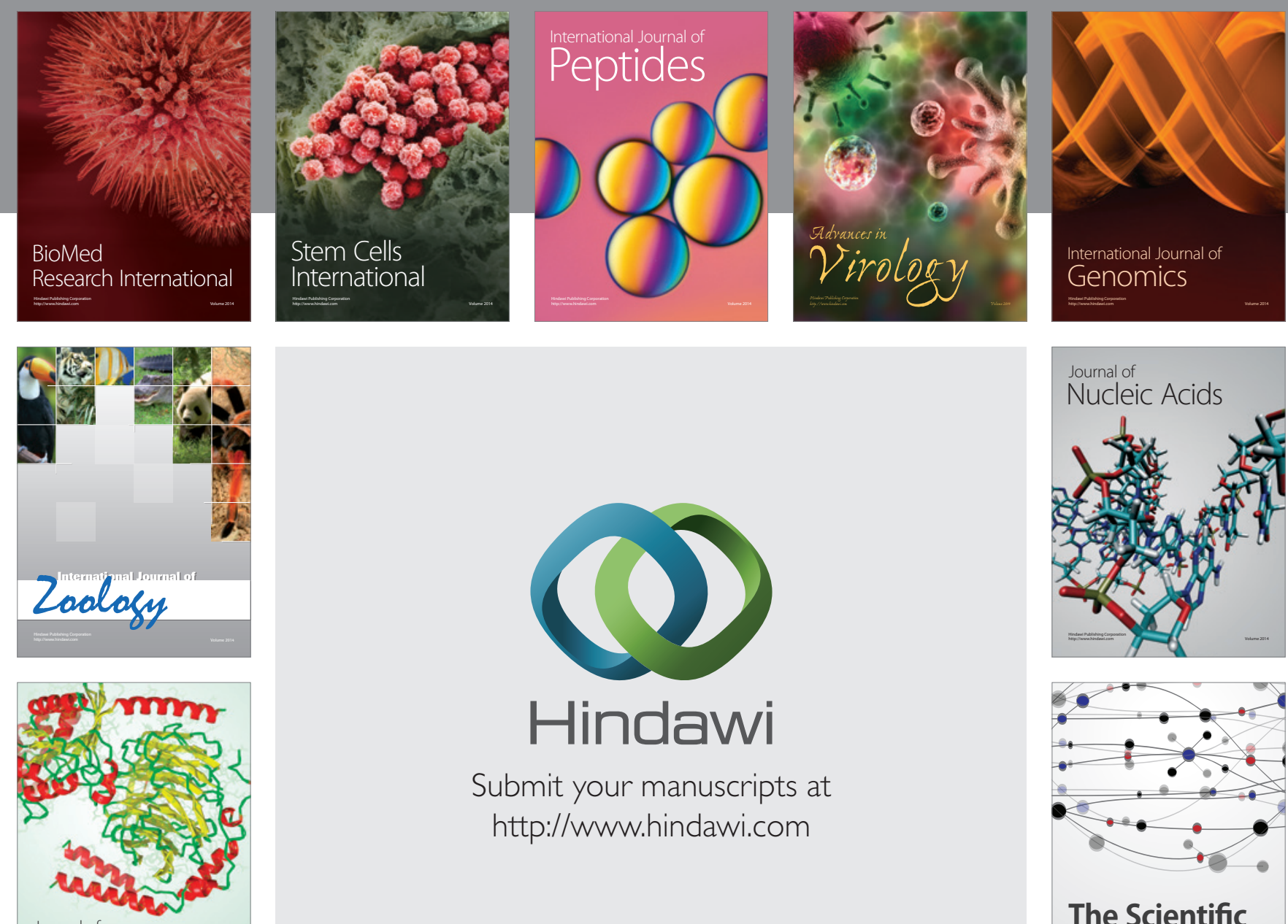

Submit your manuscripts at

http://www.hindawi.com

Journal of
Signal Transduction
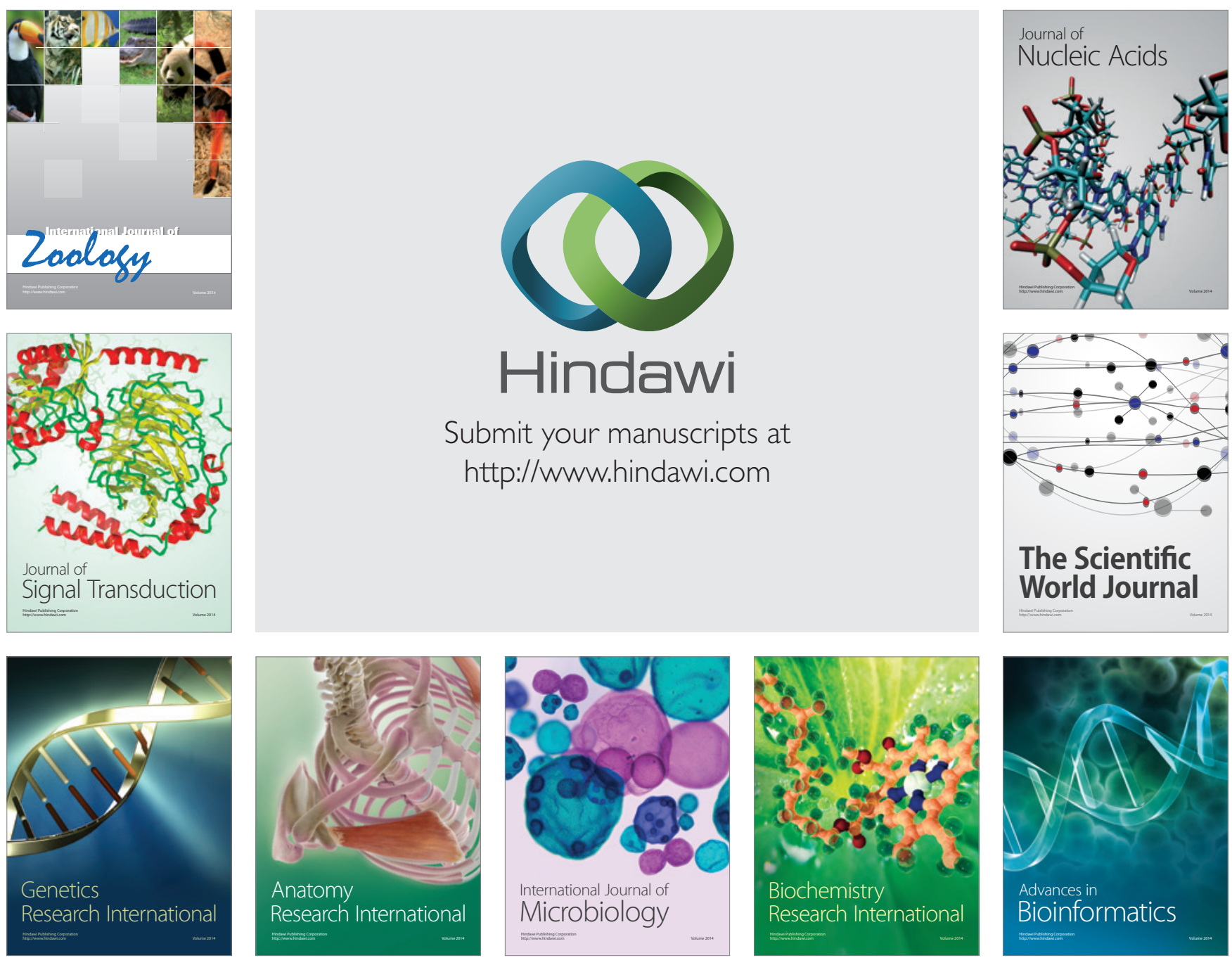

The Scientific World Journal
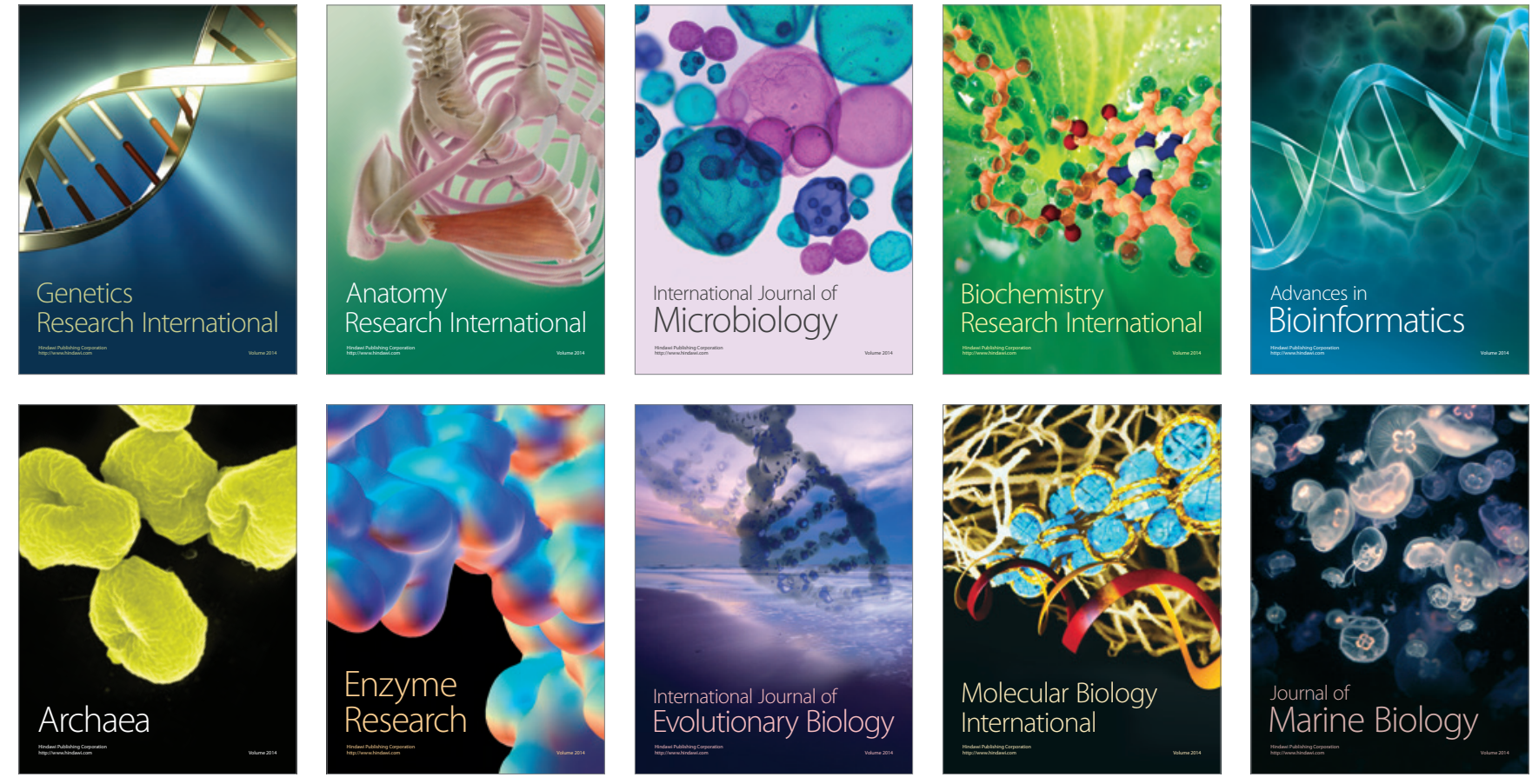